\title{
Global and local missions of cAMP signaling in neural plasticity, learning, and memory
}

\author{
Daewoo Lee* \\ Neuroscience Program, Department of Biological Sciences, Ohio University, Athens, OH, USA
}

The fruit fly Drosophila melanogaster has been a popular model to study CAMP signaling and resultant behaviors due to its powerful genetic approaches. All molecular components (AC, PDE, PKA, CREB, etc) essential for CAMP signaling have been identified in the fly. Among them, adenylyl cyclase (AC) gene rutabaga and phosphodiesterase (PDE) gene dunce have been intensively studied to understand the role of CAMP signaling. Interestingly, these two mutant genes were originally identified on the basis of associative learning deficits. This commentary summarizes findings on the role of cAMP in Drosophila neuronal excitability, synaptic plasticity and memory. It

\section{OPEN ACCESS}

Edited by: Apostolos Zarros,

University of Glasgow, UK

Reviewed by:

Robin L. Cooper,

University of Kentucky, USA

Nikolina Skandali,

University of Cambridge, UK

*Correspondence:

Daewoo Lee,

Neuroscience Program,

Department of Biological Sciences,

Ohio University, Athens,

$\mathrm{OH}$ 45701, USA

leed1@ohio.edu

Specialty section:

This article was submitted to Experimental Pharmacology

and Drug Discovery,

a section of the journal

Frontiers in Pharmacology

Received: 29 May 2015

Accepted: 20 July 2015

Published: 04 August 2015

Citation:

Lee D (2015) Global and local missions of CAMP signaling in neural

plasticity, learning, and memory.

Front. Pharmacol. 6:161.

doi: 10.3389/fphar.2015.00161 mainly focuses on two distinct mechanisms (global versus local) regulating excitatory and inhibitory synaptic plasticity related to cAMP homeostasis. This dual regulatory role of CAMP is to increase the strength of excitatory neural circuits on one hand, but to act locally on postsynaptic GABA receptors to decrease inhibitory synaptic plasticity on the other. Thus the action of cAMP could result in a global increase in the neural circuit excitability and memory. Implications of this CAMP signaling related to drug discovery for neural diseases are also described.

\section{Keywords: Drosophila melanogaster, synaptic plasticity, associative learning and memory, rutabaga, dunce, cAMP homeostasis}

Since its discovery in 1958 (Sutherland, 1992; Pittenger et al., 2012), the cyclic AMP signaling pathway has been shown to regulate a plethora of cellular functions including energy metabolism, gene expression, development, apoptosis and exocytosis. This second messenger molecule is synthesized from ATP by a family of enzymes called adenylyl cyclases (ACs), activated by Gprotein coupled receptors (GPCRs). Considering the importance of this intracellular signaling, it is not surprising that cAMP levels in the cell are negatively regulated by another group of enzymes - phosphodiesterases (PDEs). An increase in cAMP levels activates protein kinase A (PKA) which then phosphorylates target proteins including other kinases, transcriptional factors and ion channels. Therefore, its effects can be achieved by short- or long-term fashion. The latter is a protein synthesis-dependent process and thus mediated by a well-known transcription factor cAMP response element-binding protein (CREB).

Many isoforms of ACs and PDEs have been identified in the nervous system, indicating critical roles of cAMP in neural function (Pittenger et al., 2012). Indeed, cAMP signaling in the brain is known to mediate numerous neural processes from development, cellular excitability, synaptic plasticity, learning and memory, pain and motor function to neurodegeneration and drugs of abuse (Pierre et al., 2009; Bollen and Prickaerts, 2012; Kandel, 2012; Pittenger et al., 2012). In this commentary, I will specifically focus on the role of cAMP signaling in neural excitability, synaptic plasticity, learning and memory. Other important functions of cAMP signaling in the nervous system 
can be found in several outstanding reviews elsewhere (refer to Pittenger et al., 2012).

The cAMP signaling pathway mediates synaptic plasticity in both vertebrates and invertebrates. In a sea slug Aplysia, it was demonstrated that cAMP signaling mediates short- and long-term facilitation (LTF) at sensorimotor synapses (Brunelli et al., 1976; Schacher et al., 1988; Kaang et al., 1993; Bartsch et al., 1995; Kandel, 2012). The LTF was dependent on the action of CREB and new protein synthesis, and subsequently shown to mediate memory formation in Aplysia. In the rodent hippocampus, cAMP is involved in long-term potentiation (LTP) at excitatory glutamatergic synapses (Frey et al., 1993; Weisskopf et al., 1994; Silva et al., 1998). All these findings support the idea that cAMP-dependent synaptic plasticity is responsible for behavioral learning and memory at the whole organism level in both invertebrates and vertebrates. The fruit fly Drosophila melanogaster has been widely used to study molecular and cellular mechanisms of learning and memory due to its sophisticated genetic approaches. In addition, Drosophila nervous system contains all molecular components (e.g., AC, PDE, PKA, CREB, etc) essential for the CAMP signaling pathway. Drosophila AC gene rutabaga and PDE gene dunce have been intensively studied to understand the role of cAMP signaling in the nervous system. These two mutant genes were originally identified on the basis of associative learning deficits (Dudai et al., 1976; Chen et al., 1986; Levin et al., 1992; Busto et al., 2010). Further, the cAMP signaling pathway in the fly regulates synaptic plasticity at both peripheral neuromuscular junction (NMJ) as well as central synapses, where alterations in facilitation and post-tetanic potentiation were observed in mutant flies (dunce and rutabaga) with defects in cAMP signaling (Zhong and Wu, 1991; Cheung et al., 1999; Lee and O'Dowd, 2000; Ganguly and Lee, 2013). All these findings strongly support that Drosophila is an excellent model system to study the role of cAMP signaling in synaptic plasticity and the resultant behavior-learning and memory.

In this mini review, I summarize the findings on the role of cAMP in Drosophila neuronal excitability, synaptic plasticity, and learning and memory. The main emphasis is to understand distinct mechanisms (global versus local) regulating excitatory and inhibitory synaptic transmission related to cAMP homeostasis. At the end, I comment on implications of this research on disease therapy.

\section{Global Missions of cAMP Signaling}

\section{Neuronal Excitability}

One common effect of cAMP on neural function is to modulate cellular excitability. In an Aplysia sensory neuron, cAMP increased spike duration and excitability (Goldsmith and Abrams, 1992). Excitability of the rodent hippocampal neurons was also increased by cAMP signaling which subsequently enhances LTP (Gruart et al., 2012). Further, striatal neuronal excitability has been shown to be regulated by cAMP signaling (Threlfell and West, 2013).

In Drosophila NMJ, the excitability of a motor neuron is regulated by cAMP signaling (Zhong and $\mathrm{Wu}, 1991,2004)$. This change increases the excitability of presynaptic terminals, thus influencing release of neurotransmitter (NT) glutamate. This cAMP effect on excitability was also observed in Drosophila central nervous system (CNS). In contrast to mammalian CNS, acetylcholine is the primary excitatory NT in Drosophila CNS (Restifo and White, 1990; Lee and O'Dowd, 1999). These cholinergic neurons play a critical role in almost all higher brain function in Drosophila as glutamatergic neurons do in mammalian CNS. Due to difficulties in recording electrical signals from a single neuron in the fly brain, Drosophila primary neuronal culture has become a good alternative to study ionic and synaptic currents from central neurons (O'Dowd, 1995; Lee and O'Dowd, 1999; Ganguly and Lee, 2013). When fly cholinergic neurons were focally exposed to a popular AC activator forskolin (FSK), action potential (AP) frequency was drastically increased. GABAergic neurons also showed an increase in AP in response to the focal application of FSK (Ganguly and Lee, 2013). This change is a direct effect of cAMP as excitatory cholinergic inputs to GABAergic neurons were blocked by an acetylcholine receptor (AChR) blocker curare. In Drosophila, live cholinergic and GABAergic neurons can be easily identified using a live fluorescent marker (e.g., RFP or GFP; Wiemerslage et al., 2013). Increased excitability by CAMP is primarily achieved through PKA, which mediates a phosphorylation-induced reduction in potassium channel conductance (Wright and Zhong, 1995; Delgado et al., 1998). Taken together, all these findings show that cAMP-PKA signaling directly enhanced the excitability of all types of neurons-not only inhibitory GABAergic (Ganguly and Lee, 2013) but also excitatory cholinergic and glutamatergic neurons (Zhong and Wu, 1991, 2004; Lee and O'Dowd, 2000). Further, these findings indicate that cAMP signaling increases pre-synaptic NT release through enhanced excitability.

\section{Synaptic Plasticity in CNS}

Synaptic transmission is a primary way to communicate between neurons in the brain. The synapse is not static, rather dynamically changes its strength which is known as synaptic plasticity, an important subcellular mechanism underlying learning and memory (Bhalla and Iyengar, 1999; Kandel, 2001). A key molecule involved in this plasticity is the second messenger cAMP.

The cAMP-PKA pathway is known to regulate synaptic plasticity (e.g., LTP) in the mossy fibers and CA3 pyramidal cells in hippocampal slices (Nicoll and Malenka, 1995; Silva et al., 1998). The cAMP signaling pathway has been shown to increase the pre-synaptic vesicle release probability (Chen and Regehr, 1997), through enhanced vesicle docking before exocytosis (Sudhof, 2004). In Aplysia, PKA increases an influx of $\mathrm{Ca}^{2+}$ into the pre-synaptic neuron, facilitates vesicle fusion, and glutamate release resulting in short term facilitation by inhibiting the S-type $\mathrm{K}^{+}$channels (Kandel, 2001). Studies in Drosophila NMJ have shown that cAMP alters pre-synaptic release probability and facilitation (Zhong and Wu, 1991), which can be achieved by the reduction of $\mathrm{K}^{+}$currents (Zhong et al., 1992) or by activation of hyperpolarization-activated cyclic nucleotide-gated (HCN) channels (Cheung et al., 2005). We have demonstrated that CAMP signaling can regulate functional plasticity, independent of differentiation, at excitatory cholinergic synapses between cultured Drosophila neurons (Lee and O'Dowd, 


\section{A}
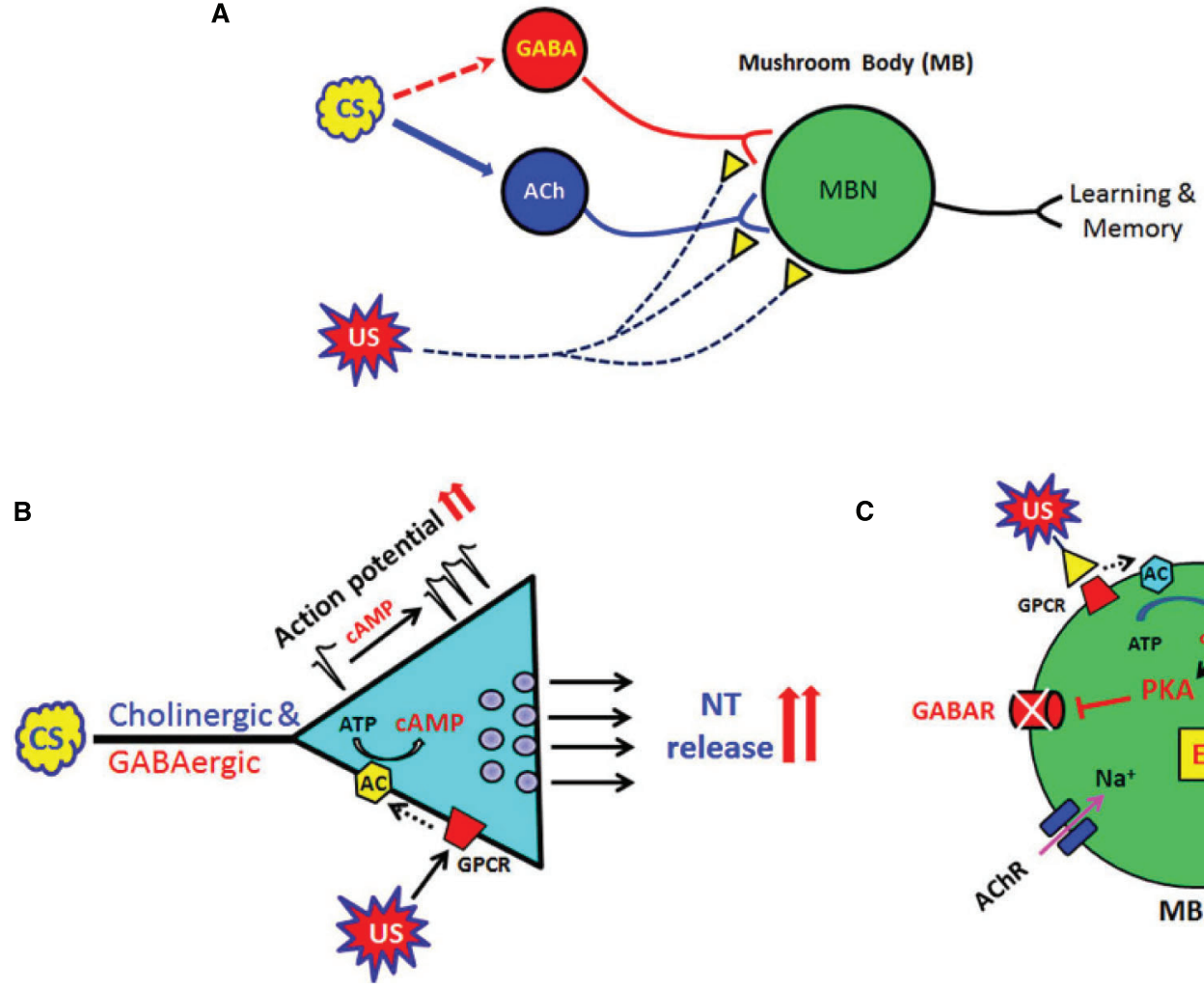

C

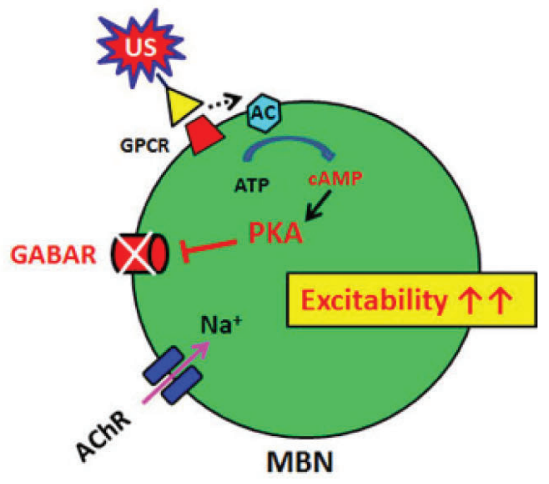

FIGURE 1 | Global and local actions of cAMP signaling in neural plasticity, learning and memory in Drosophila. (A) A diagram showing Drosophila neural circuits which mediate associative learning and memory formation. Sensory information (CS) is mainly transduced through excitatory cholinergic synaptic inputs to the fly learning and memory center, mushroom body (MB). Inhibitory GABAergic inputs to MB are known to shape this sensory information transduction although its sensory processing circuits remain to be explored (dotted arrow). US (reward or punishment) is mediated through modulatory synaptic inputs such as dopaminergic or serotonergic. Association of the CS and US in MB is the basis of Drosophila learning. (B) Cyclic AMP has global (or general) roles to increase presynaptic excitability and neurotransmitter release. These changes are found in all central neurons including excitatory cholinergic and inhibitory GABAergic neurons. (C) In contrast, cAMP suppresses ionotropic GABA receptors in the postsynaptic neuron and thus enhances overall excitability in MB neural circuits. Acetylcholine receptors (AChRs) in Drosophila postsynaptic neurons were not affected by cAMP signaling (Lee and O'Dowd, 2000). Conditional stimulus (CS); MB neurons (MBN); unconditional stimulus (US).
2000). Presynaptic GABA release was also greatly increased by an AC activator FSK (Ganguly and Lee, 2013). Further, it has been shown that facilitation is impaired in cAMP signaling mutants (Zhao and Wu, 1997; Lee and O'Dowd, 2000; Ganguly and Lee, 2013). All the findings show that the cAMP-PKA pathway plays an important role in regulating plasticity at excitatory as well as inhibitory synapses in Drosophila CNS. Therefore, global actions of cAMP signaling on synaptic plasticity appear to be enhanced presynaptic release of NT via increasing presynaptic excitability in all types of neurons including excitatory cholinergic and inhibitory GABAergic neurons (Figure 1).

\section{Local Mission of cAMP Signaling}

Cyclic AMP-PKA signaling generally (and globally) increases neuronal excitability and presynaptic release of NTs in Drosophila nervous system. This increase was observed not only at excitatory but also inhibitory synapses. This can be contradictory because an increase in inhibitory synaptic transmission by cAMP is expected to reduce overall strength of excitatory synaptic transmission in certain neural circuits. Therefore, we further examined the role of
cAMP signaling in inhibitory GABAergic synaptic transmission in Drosophila (Ganguly and Lee, 2013). When postsynaptic cAMP signaling was specifically blocked by a membrane-permeable PKA inhibitor, the frequency of GABAergic synaptic currents was increased by focal application of FSK like excitatory cholinergic synaptic currents (see above). However, without the blocker in a postsynaptic neuron, GABAergic synaptic transmission was suppressed by FSK even if presynaptic GABA release was increased (Ganguly and Lee, 2013). This confirmed that PKA suppresses GABAergic synaptic transmission by regulating postsynaptic GABA receptor sensitivity through phosphorylation. Among three Drosophila ionotropic GABA receptors (RDL, LCCH3, and GRD; Harvey et al., 1994; Hosie et al., 1997), the GABA resistant to dieldrin (RDL) receptor subunit is widely expressed in several regions of the Drosophila brain (Harrison et al., 1996) and its expression in the fly learning and memory center mushroom body (MB) is inversely correlated to olfactory learning (Liu et al., 2007). Based on the observation that RDL containing GABA receptors mediate the majority of GABAergic synaptic currents in Drosophila (Lee et al., 2003; Ganguly and Lee, 2013), the action of cAMP on GABAergic synaptic 
currents is likely through the GABA RDL subunit. PKA-mediated phosphorylation of RDL subunits and subsequent GABA receptor internalization may occur specifically (and locally) in the postsynaptic region (Mou et al., 2011; Vithlani et al., 2011).

Drosophila brain has shown the presence of a large number of inhibitory GABAergic interneurons, some of which innervate into the MB (Yasuyama et al., 2002; Busto et al., 2010). These interneurons have been shown to be important for various forms of information processing and behaviors including learning and memory (Olsen and Wilson, 2008; Isaacson and Scanziani, 2011; Wilson, 2013). Strengthening in the efficacy of excitatory transmission causes enhanced neural circuit plasticity. Therefore, the suppression of inhibitory transmission by a common second messenger like cAMP is expected to further increase the neural circuit excitability. In Drosophila, cAMP-PKA signaling increases excitability at the cholinergic synapses (Yuan and Lee, 2007) but decreases the conductance of postsynaptic GABA receptors (Ganguly and Lee, 2013). These findings demonstrate a novel dual regulatory role of cAMP by showing that it increases overall presynaptic function globally, but acts locally on postsynaptic GABA receptors to decrease GABAergic plasticity. Thus the action of cAMP results in further increases in neural excitability (Figure 1).

\section{Cyclic AMP Homeostasis, Neural Circuits, Learning, and Memory}

A number of Drosophila mutants showing defects in the cAMPsignaling cascade were originally identified on the basis of associative learning deficits (Dudai et al., 1976; Livingstone et al., 1984; Chen et al., 1986; Levin et al., 1992). Among them, a Drosophila $\mathrm{Ca}^{2+} / \mathrm{CaM}$-dependent AC rutabaga is known to function as a coincidence detector during learning and memory consolidation (Tomchik and Davis, 2009; Gervasi et al., 2010). A typical associative learning task is comprised of two different stimuli-conditional (CS; sensory input such as smell or visual) and unconditional stimuli (US; reward or punishment). In Drosophila, excitatory cholinergic and GABAergic inputs are considered to deliver CS signals while a variety of synaptic modulators (e.g., dopamine, serotonin, etc) are involved in the transduction of US signals (Figure 1). The US modulators typically stimulate GPCR to activate AC while synaptic inputs from CS neural circuits increase cellular excitability and also $\mathrm{Ca}^{2+} / \mathrm{CaM}$. Therefore, AC serves as a coincidence detector of CS and US. A Drosophila PDE4 homolog dunce is also known to cause defects in short-term memory (Dudai et al., 1976; Chen et al., 1986). Both rutabaga and dunce enzymes in Drosophila show changes in the strength of Drosophila excitatory synapses (Zhong and Wu, 1991; Lee and O'Dowd, 2000) as well as inhibitory GABAergic synapses (Ganguly and Lee, 2013). Therefore, cAMPdependent synaptic plasticity must be an essential feature for neural circuits in mediating learning and memory.

Interestingly, consequences of rutabaga and dunce mutants are opposite in terms of intracellular cAMP levels. The former decreases basal cAMP levels and thus does not able to temporally increase cAMP in response to acute stimulation, while the letter increases the basal level of intracellular cAMP much more than that in wild type. However, both mutations cause defects in synaptic plasticity and memory, strongly indicating the importance of cAMP homeostasis. Proper regulation of intracellular cAMP appears to be critical for neural plasticity and memory in fly. Thus it is of interest to understand the role of these two enzymes maintaining homeostasis of cAMP in Drosophila neurons.

The Drosophila MB is comprised of several subdivisions (e.g., $\alpha, \beta, \gamma$ lobe, etc) (Keene and Waddell, 2007; Guven-Ozkan and Davis, 2014). Different MB neurons are involved in distinct types and/or phases of associative learning and memory in the fly. Further details can be found in several excellent reviews on $\mathrm{MB}$ structure and function (Fahrbach, 2006; Waddell, 2013; GuvenOzkan and Davis, 2014). The synaptic inputs to MB are mainly coming from excitatory cholinergic and GABAergic neurons from the centers of sensory system (e.g., antennal lobes for olfaction) as they primarily mediate sensory information. For example, projection neurons (PNs) in antennal lobes relay sensory signals to $\mathrm{MB}$ neurons for olfactory associative learning. PN neurons are cholinergic and thus excitatory synaptic inputs. MB neurons also receive synaptic inputs from GABAergic neurons mainly from the region called lateral horn (LH; Busto et al., 2010). Since rutabaga and dunce are preferentially expressed in MB, cAMP signaling is important for synaptic plasticity in MB neurons and also essential for learning and memory.

Learning and memory defects in rutabaga can be easily explained as its basal cAMP level is too low so that no neural input signal can induce proper cAMP-dependent synaptic plasticity mediating learning memory. Interestingly, dunce mutants with high levels of cAMP in MB neurons also show defects in short-term memory (Gervasi et al., 2010). Further, the dunce $\mathrm{MB}$ neurons show an increase in PKA levels. These findings suggest that cAMP-mediated potentiation of cholinergic synaptic transmission and inhibition of GABA receptor should be greater in dunce neurons. However, the dunce and rutabaga mutants, despite having opposing effects on cellular cAMP levels, showed very similar defects in synaptic plasticity at both excitatory and inhibitory synapses (Lee and O'Dowd, 2000; Ganguly and Lee, 2013). Several other studies have also shown that dunce and rutabaga have similar defects in growth cone motility, neural plasticity and more importantly, short-term memory (Kim and Wu, 1996; Gasque et al., 2006). Although the effects of cAMP on cholinergic and GABAergic synaptic plasticity in dunce and rutabaga mutants are similar, it is very likely that the molecular mechanisms underlying these responses differ in the two mutants. It has been shown that increased PKA activity in mouse hippocampus hyper-phosphorylates several downstream molecular targets including a tyrosine phosphatase, correlates with decreased PDE protein levels and results in memory defects (Giralt et al., 2011). Therefore, it is expected that high basal levels of cAMP due to the dunce mutation leads to the activation of phosphatase(s) and thus reduces the effects of cAMP. However, this remains to be explored in Drosophila nervous system. Taken together, all these findings strongly suggest that disruption of cellular cAMP homeostasis can alter excitatory cholinergic and inhibitory GABAergic synaptic plasticity and hence cause defects 
in associative learning, although the underlying mechanisms leading to this effect can be different (e.g., reduced PKA activity in rutabaga versus increased phosphatase activity in dunce).

\section{Drug Targets in cAMP Signaling}

Cyclic AMP is the most abundant and important second messenger in the nervous system. Therefore, it makes sense that its signaling involves a variety of physiological and pathological processes such as learning and memory, pain, drug addiction and neurodegeneration. Molecular components in cAMP-PKA signaling pathway should be excellent targets for the development of new therapeutic strategies. These components in cAMP signaling are very well conserved throughout animal groups and, therefore, findings from Drosophila can be directly implicated in mammalian systems including human.

Memory is an important physiological process for survival and better quality of life. Thus any compromise in this behavior is likely a problem as seen in Alzheimer's disease (AD) and mental retardation. Given the importance of cAMP signaling in neural plasticity and cognition, any molecular component in this signaling pathway can be a potential target for drug development to enhance cognition. Particularly two enzymes have drawn more attention. The first target is a PDE which down-regulates cAMP-PKA signaling. Therefore, PDE inhibitors will prolong cAMP signaling and produce higher levels potentially enhancing cognition. Indeed, a PDE4 inhibitor HT-0712 has been shown to improve hippocampus-mediated memory in mice (Peters et al., 2014). HT-0712 also increases CRE-mediated gene expression and ameliorates spatial memory impairment in aged mice, therefore, it could be used to treat age-associated memory impairment (AAMI) in humans. In fact, clinical studies with this drug have been carried out and show significant effects on long-term memory in AAMI patients (refer to: www.dartneuroscience.com). Since an increase of cAMP-specific PDE mRNAs was observed in early stages of AD (Bollen and Prickaerts, 2012), PDE inhibitors can be used to slow/treat this disease. However, it should be kept in mind that the chronic increase of basal cAMP by PDE inhibitors can give negative impacts on learning and memory as seen in the dunce mutants.

The second promising drug target for cAMP signaling is AC. AC1/AC8 double knockout mice showed loss of LTP as well as memory (Wong et al., 1999). Further, beta amyloid peptides interfere with AC-dependent LTP in hippocampus (Yamamoto et al., 1997). Findings with rutabaga fly mutants are consistent as these flies showed defects in synaptic plasticity and short-term memory. Therefore, drugs that stimulate AC activities can be useful to treat memory deficits although no drug is on a clinical test yet. AC is an attractive drug target to be further explored.

Additional potential targets in the cAMP signaling pathway are PKA and CREB-binding protein (CBP). Rubinstein-Taybi syndrome (RTS), a genetic disorder showing mental retardation and physical abnormalities (Bourtchouladze et al., 2003) is known to be caused by mutations of CBP. Regarding the role of CREB and CBP in the nervous system, it is noteworthy to mention that some addictive drugs (e.g., amphetamine, opiates) also alter CREB expression in several brain areas (e.g., nucleus accumbens and hippocampus; Robbins et al., 2007; Nestler, 2013) indicating involvement of cAMP signaling in drug addiction. Interestingly, Drosophila has been used as a favorable model animal to study actions of addictive drugs such as cocaine and alcohol (Kaun et al., 2012). A recent study showed that Drosophila rutabaga in $\mathrm{MB}$ neurons is necessary for robust ethanol self-administration (Xu et al., 2012) demonstrating the role of cAMP signaling in reinforced behaviors. Therefore, PKA and CREB-related proteins can be excellent drug targets to treat not only cognitive deficits but also drug addiction.

Given the suitability of multiple drug targets in cAMP signaling, one important question is the therapeutic safety in addition to drug potency. In other words, how can we deliver a drug to a specific target in order to minimize toxic side effects? Chemogenetic tools have been developed to enhance specific drug delivery spatially and temporally (Sternson and Roth, 2014). One such tool is designer receptors exclusively activated by designer drugs (DREADDs), which can increase or decrease intracellular cAMP levels depending on the receptor type by a biologically inert chemical (Becnel et al., 2013). Using this tool, cAMP signaling can be regulated in a specific set of neurons (e.g., hippocampus, $\mathrm{MB})$ as well as duration of action (e.g., acute versus chronic). Therefore, CREB-mediated long-term effects can be also induced. In addition, DREADD can be an excellent research tool to uncover more specific roles of cAMP signaling in a variety of neuronal processes including learning and memory.

\section{Conclusion}

In this commentary, I focused on the role of cAMP signaling in neural excitability, synaptic plasticity, learning and memory. On the basis of work from Drosophila, dual regulatory roles (global versus local) of cAMP signaling are to increase the strength of excitatory neural circuits on one hand, but to act locally on postsynaptic GABA receptors to decrease inhibitory synaptic plasticity on the other. Thus the action of cAMP could result in a global increase in the neural circuit excitability and memory. The cAMP signaling is also implicated in pain processing, $\mathrm{AD}$ and drug addiction. All these normal and disease-related behaviors are mediated through interaction between specific neural circuits comprised of excitatory and inhibitory synapses. Therefore, knowledge gained from the studies of cAMP signaling can contribute to the development of new or more effective drugs. Since molecular components and functions of cAMP signaling pathway have been well conserved in Drosophila, it could be a useful animal model to study mechanisms underlying behaviors mediated by cAMP signaling at the molecular, physiological and circuit levels. The fly can also be an excellent drug discovery platform for diseases with defects in cAMP signaling.

\section{Acknowledgments}

This work was partially supported by the International Collaboration Grant from Korea Institute of Science and Technology (Brain Science Institute), Seoul, Korea. The author thanks R. A. Colvin for valuable inputs on previous versions of this manuscript. 


\section{References}

Bartsch, D., Ghirardi, M., Skehel, P. A., Karl, K. A., Herder, S. P., Chen, M., et al. (1995). Aplysia CREB2 represses long-term facilitation: relief of repression converts transient facilitation into long-term functional and structural change. Cell 83, 979-992. doi: 10.1016/0092-8674(95)90213-9

Becnel, J., Johnson, O., Majeed, Z. R., Tran, V., Yu, B., Roth, B. L., et al. (2013). DREADDs in Drosophila: a pharmacogenetic approach for controlling behavior, neuronal signaling, and physiology in the fly. Cell Rep. 4, 1049-1059. doi: 10.1016/j.celrep.2013.08.003

Bhalla, U. S., and Iyengar, R. (1999). Emergent properties of networks of biological signaling pathways. Science 283, 381-387. doi: 10.1126/science.283.5400.381

Bollen, E., and Prickaerts, J. (2012). Phosphodiesterases in neurodegenerative disorders. IUBMB Life 64, 965-970. doi: 10.1002/iub.1104

Brunelli, M., Castellucci, V., and Kandel, E. R. (1976). Synaptic facilitation and behavioral sensitization of the gill withdrawal reflex in Aplysia: possible role of serotonin and cyclic AMP. Science 194, 1178-1181. doi: 10.1126/science.186870

Bourtchouladze, R., Lidge, R., Catapano, R., Stanley, J., Gossweiler, S., Romashko, D., et al. (2003). A mouse model of Rubinstein-Taybi syndrome: defective longterm memory is ameliorated by inhibitors of phosphodiesterase 4. Proc. Natl. Acad. Sci. U.S.A. 100, 10518-10522. doi: 10.1073/pnas.1834280100

Busto, G. U., Cervantes-Sandoval, I., and Davis, R. L. (2010). Olfactory learning in Drosophila. Physiology 25, 338-346. doi: 10.1152/physiol.00026.2010

Chen, C.-N., Denomen, S., and Davis, R. L. (1986). Molecular analysis of cDNA clones and the corresponding genomic encoding sequences of the Drosophila dunce gene, the structural gene for cAMP phosphodiesterase. Proc. Natl. Acad. Sci. U.S.A. 83, 9313-9317. doi: 10.1073/pnas.83.24.9313

Chen, C., and Regehr, W. G. (1997). The mechanism of cAMP-mediated enhancement at a cerebellar synapse. J. Neurosci. 17, 8687-8694.

Cheung, U., Atwood, H. L., and Zucker, R. S. (2005). Presynaptic effectors contributing to cAMP-induced synaptic potentiation in Drosophila. J. Neurobiol. 66, 273-280. doi: 10.1002/neu.20218

Cheung, U. S., Shayan, A. J., Boulianne, G. L., and Atwood, H. L. (1999). Drosophila larval neuromuscular junction's responses to reduction of cAMP in the nervous system. J. Neurobiol. 40, 1-13.

Delgado, R., Davis, R., Bono, M. R., Latorre, R., and Labarca, P. (1998). Outward currents in Drosophila larval neurons: dunce lacks a maintained outward current component downregulated by cAMP. J. Neurosci. 18, 1399-1407.

Dudai, Y., Jan, Y. N., Byers, D., Quinn, W. G., and Benzer, S. (1976). dunce, a mutant of Drosophila deficient in learning. Proc. Natl. Acad. Sci. U.S.A. 73, 1684-1688. doi: 10.1073 /pnas.73.5.1684

Fahrbach, S. E. (2006). Structure of the mushroom bodies of the insect brain. Annu. Rev. Entomol. 51, 209-232. doi: 10.1146/annurev.ento.51.110104.150954

Frey, U., Huang, Y. Y., and Kandel, E. R. (1993). Effects of cAMP simulate a late stage of LTP in hippocampal CA1 neurons. Science 260, 1661-1664. doi: $10.1126 /$ science. 8389057

Ganguly, A., and Lee, D. (2013). Suppression of inhibitory GABAergic transmission by cAMP signaling pathway: alterations in learning and memory mutants. Eur. J. Neurosci. 37, 1383-1393. doi: 10.1111/ejn.12144

Gasque, G., Labarca, P., Delgado, R., and Darszon, A. (2006). Bridging behavior and physiology: ion channel perspective on mushroom body-dependent olfactory learning and memory in Drosophila. J. Cell. Physiol. 209, 1046-1053. doi: 10.1002/jcp.20764

Gervasi, N., Tchenio, P., and Preat, T. (2010). PKA dynamics in a Drosophila learning center: coincidence detection by rutabaga adenylyl cyclase and spatial regulation by dunce phosphodiesterase. Neuron 65, 516-529. doi: 10.1016/j.neuron.2010.01.014

Giralt, A., Saavedra, A., Carreton, O., Xifro, X., Alberch, J., and Perez-Navarro, E. (2011). Increased PKA signaling disrupts recognition memory and spatial memory: role in Huntington's disease. Hum. Mol. Genet. 20, 4232-4247. doi: 10.1093/hmg/ddr351

Goldsmith, B. A., and Abrams, T. W. (1992). cAMP modulates multiple $\mathrm{K}^{+}$currents, increasing spike duration and excitability in Aplysia sensory neurons. Proc. Natl. Acad. Sci. U.S.A. 89, 11481-11485. doi: 10.1073/pnas.89.23.11481

Gruart, A., Benito, E., Delgado-García, J. M., and Barco, A. (2012). Enhanced cAMP response element-binding protein activity increases neuronal excitability, hippocampal long-term potentiation, and classical eyeblink conditioning in alert behaving mice. J. Neurosci. 32, 17431-17441. doi: 10.1523/JNEUROSCI.4339-12.2012
Guven-Ozkan, T., and Davis, R. L. (2014). Functional neuroanatomy of Drosophila olfactory memory formation. Learn. Mem. 21, 519-526. doi: 10.1101/lm.034363.114

Harrison, J. B., Chen, H. H., Sattelle, E., Barker, P. J., Huskisson, N. S., Rauh, J. J., et al. (1996). Immunocytochemical mapping of a C-terminus antipeptide antibody to the GABA receptor subunit, RDL in the nervous system in Drosophila melanogaster. Cell Tissue Res. 284, 269-278. doi: 10.1007/s004410050587

Harvey, R. J., Schmitt, B., Hermans-Borgmeyer, I., Gundelfinger, E. D., Betz, H., and Darlison, M. G. (1994). Sequence of a Drosophila ligand-gated ionchannel polypeptide with an unusual amino-terminal extracellular domain. J. Neurochem. 62, 2480-2483. doi: 10.1046/j.1471-4159.1994.62062480.x

Hosie, A. M., Aronstein, K., Sattelle, D. B., and ffrench-Constant, R. H. (1997). Molecular biology of insect neuronal GABA receptors. Trends Neurosci. 20, 578-583. doi: 10.1016/S0166-2236(97)01127-2

Isaacson, J. S., and Scanziani, M. (2011). How inhibition shapes cortical activity. Neuron 72, 231-243. doi: 10.1016/j.neuron.2011.09.027

Kaang, B. K., Kandel, E. R., and Grant, S. G. (1993). Activation of cAMPresponsive genes by stimuli that produce long-term facilitation in Aplysia sensory neurons. Neuron 10, 427-435. doi: 10.1016/0896-6273(93)90331-K

Kandel, E. R. (2001). The molecular biology of memory storage: a dialogue between genes and synapses. Science 294, 1030-1038. doi: 10.1126/science.1067020

Kandel, E. R. (2012). The molecular biology of memory: cAMP, PKA, CRE, CREB-1, CREB-2, and CPEB. Mol. Brain 5, 14. doi: 10.1186/1756-6606-5-14

Kaun, K. R., Devineni, A. V., and Heberlein, U. (2012). Drosophila melanogaster as a model to study drug addiction. Hum. Genet. 131, 959-975. doi: 10.1007/s00439012-1146-6

Keene, A. C., and Waddell, S. (2007). Drosophila olfactory memory: single genes to complex neural circuits. Nat. Rev. Neurosci. 8, 341-354. doi: 10.1038/nrn2098

Kim, Y. T., and Wu, C. F. (1996). Reduced growth cone motility in cultured neurons from Drosophila memory mutants with a defective cAMP cascade. J. Neurosci. 16, 5593-5602.

Lee, D., and O’Dowd, D. K. (1999). Fast excitatory synaptic transmission mediated by nicotinic acetylcholine receptors in Drosophila neurons. J. Neurosci. 19, 5311-5321.

Lee, D., and O'Dowd, D. K. (2000). cAMP-dependent plasticity at excitatory cholinergic synapses in Drosophila neurons: alterations in the memory mutant dunce. J. Neurosci. 20, 2104-2111.

Lee, D., Su, H., and O’Dowd, D. K. (2003). GABA receptors containing Rdl subunits mediate fast inhibitory synaptic transmission in Drosophila neurons. J. Neurosci. 23, 4625-4634.

Levin, L. R., Han, P. L., Hwang, P. M., Feinstein, P. G., Davis, R. L., and Reed, R. R. (1992). The Drosophila learning and memory gene rutabaga encodes a Ca21/calmodulin-responsive adenyl cyclase. Cell 68, 479-489. doi: 10.1016/0092-8674(92)90185-F

Liu, X., Krause, W. C., and Davis, R. L. (2007). GABAA receptor RDL inhibits Drosophila olfactory associative learning. Neuron 56, 1090-1102. doi: 10.1016/j.neuron.2007.10.036

Livingstone, M. S., Sziber, P. P., and Quinn, W. G. (1984). Loss of calcium/calmodulin responsiveness in adenylate cyclase of rutabaga, a Drosophila learning mutant. Cell 37, 205-215. doi: 10.1016/00928674(84)90316-7

Mou, L., Heldt, S. A., and Ressler, K. J. (2011). Rapid brain-derived neurotrophic factor-dependent sequestration of amygdala and hippocampal GABA(A) receptors via different tyrosine receptor kinase B-mediated phosphorylation pathways. Neuroscience 176, 72-85. doi: 10.1016/j.neuroscience.2010.12.041

Nestler, E. J. (2013). Cellular basis of memory for addiction. Dialogues Clin. Neurosci. 15, 431-443.

Nicoll, R. A., and Malenka, R. C. (1995). Contrasting properties of two forms of long-term potentiation in the hippocampus. Nature 377, 115-118. doi: $10.1038 / 377115 \mathrm{a} 0$

O’Dowd, D. K. (1995). Voltage-gated currents and firing properties of embryonic Drosophila neurons grown in a chemically defined medium. J. Neurobiol. 27, 113-126. doi: 10.1002/neu.480270111

Olsen, S. R., and Wilson, R. I. (2008). Lateral presynaptic inhibition mediates gain control in an olfactory circuit. Nature 452, 956-960. doi: 10.1038/nature06864

Peters, M., Bletsch, M., Stanley, J., Wheeler, D., Scott, R., and Tully, T. (2014). The PDE4 inhibitor HT-0712 improves hippocampus-dependent memory in aged mice. Neuropsychopharmacology 39, 2938-2948. doi: 10.1038/npp. 2014.154 
Pierre, S., Eschenhagen, T., Geisslinger, G., and Scholich, K. (2009). Capturing adenylyl cyclases as potential drug targets. Nat. Rev. Drug Discov. 8, 321-335. doi: $10.1038 / \mathrm{nrd} 2827$

Pittenger, C., Nestler, E. J., and Duman, R. S. (2012). "Cyclic nucleotides in the nervous system," in Basic Neurochemistry: Principles of Molecular, Cellular, and Medical Neurobiology, 8th Edn, eds S. T. Brady, G. J. Siegel, R. W. Albers, and D. L. Price (New York: Academic Press), 423-441.

Restifo, L. L., and White, K. (1990). Molecular and genetic approaches to neurotransmitter and neuromodulator systems in Drosophila. Adv. Insect. Physiol. 22, 115-219. doi: 10.1016/S0065-2806(08)60006-5

Robbins, T., Cardinal, R. N., DiCiano, P., Halligan, P. W., Hellemans, K., Lee, J., et al. (2007). "Neuroscience of drugs and addiction," in Drugs and the Future: Brain Science, Addiction and Society, eds D. Nutt, T. W. Robbins, G. V. Stimson, M. Ince, and A. Jackson (New York: Elsevier Inc.), 11-87. doi: 10.1016/b978-0123706249/50006-2

Schacher, S., Castellucci, V. F., and Kandel, E. R. (1988). cAMP evokes long-term facilitation in Aplysia sensory neurons that requires new protein synthesis. Science 240, 1667-1669. doi: 10.1126/science.2454509

Silva, A. J., Kogan, J. H., Frankland, P. W., and Kida, S. (1998). CREB and memory. Annu. Rev. Neurosci. 21, 217-148. doi: 10.1146/annurev.neuro.21.1.127

Sternson, S. M., and Roth, B. L. (2014). Chemogenetic tools to interrogate brain functions. Ann. Rev. Neurosci. 37, 387-407. doi: 10.1146/annurev-neuro071013-014048

Sudhof, T. C. (2004). The synaptic vesicle cycle. Annu. Rev. Neurosci. 27, 509-547. doi: 10.1146/annurev.neuro.26.041002.131412

Sutherland, E. W. (1992). "Studies on the mechanism of hormone action," in Nobel Lectures in Physiology or Medicine (1971-1980), ed. J. Lindsten (Singapore: World Scientific Publishing Co), 1-22.

Threlfell, S., and West, A. R. (2013). Modulation of striatal neuron activity by cyclic nucleotide signaling and phosphodiesterase inhibition. Basal Ganglia 3, 137-146. doi: 10.1016/j.baga.2013.08.001

Tomchik, S. M., and Davis, R. L. (2009). Dynamics of learning-related cAMP signaling and stimulus integration in the Drosophila olfactory pathway. Neuron 64, 510-521. doi: 10.1016/j.neuron.2009.09.029

Vithlani, M., Terunuma, M., and Moss, S. J. (2011). The dynamic modulation of $\mathrm{GABA}(\mathrm{A})$ receptor trafficking and its role in regulating the plasticity of inhibitory synapses. Physiol. Rev. 91, 1009-1022. doi: 10.1152/physrev.00015.2010

Waddell, S. (2013). Reinforcement signalling in Drosophila; dopamine does it all after all. Curr. Opin. Neurobiol 3, 324-329. doi: 10.1016/j.conb.2013.01.005

Weisskopf, M. G., Castillo, P. E., Zalutsky, R. A., and Nicoll, R. A. (1994). Mediation of hippocampal mossy fiber long-term potentiation by cyclic AMP. Science 265, 1878-1882. doi: $10.1126 /$ science.7916482

Wiemerslage, L., Schultz, B. J., Ganguly, A., and Lee, D. (2013). Selective degeneration of dopaminergic neurons by $\mathrm{MPP}^{+}$and its rescue by D2 autoreceptors in Drosophila primary culture. J. Neurochem. 126, 529-540. doi: $10.1111 /$ jnc. 12228
Wilson, R. I. (2013). Early olfactory processing in Drosophila: mechanisms and principles. Annu. Rev. Neurosci. 36, 217-241. doi: 10.1146/annurev-neuro062111-150533

Wong, S. T., Athos, J., Figueroa, X. A., Pineda, V. V., Schaefer, M. L., Chavkin, C. C., et al. (1999). Calcium-stimulated adenylyl cyclase activity is critical for hippocampus-dependent long-term memory and late phase LTP. Neuron 23, 787-798. doi: 10.1016/S0896-6273(01)80036-2

Wright, N. J., and Zhong, Y. (1995). Characterization of $\mathrm{K}^{+}$currents and the cAMP-dependent modulation in cultured Drosophila mushroom body neurons identified by lacZ expression. J. Neurosci. 15, 1025-1034.

Yamamoto, M., Ozawa, H., Saito, T., Hatta, S., Riederer, P., and Takahata, N. (1997). $\mathrm{Ca}^{2+} / \mathrm{CaM}$-sensitive adenylyl cyclase activity is decreased in the Alzheimer's brain: possible relation to type I adenylyl cyclase. J. Neural Transm 104, 721-732. doi: 10.1007/BF01291889

Yasuyama, K., Meinertzhagen, I. A., and Schurmann, F. W. (2002). Synaptic organization of the mushroom body calyx in Drosophila melanogaster. J. Comp. Neurol. 445, 211-226. doi: 10.1002/cne.10155

Yuan, N., and Lee, D. (2007). Suppression of excitatory cholinergic synaptic transmission by Drosophila dopamine D1-like receptors. Eur. J. Neurosci. 26, 2417-2427. doi: 10.1111/j.1460-9568.2007.05870.x

Xu, S., Chan, T., Shah, V., Zhang, S., Pletcher, S. D., and Roman, G. (2012). The propensity for consuming ethanol in Drosophila requires rutabaga adenylyl cyclase expression within mushroom body neurons. Genes Brain Behav. 11, 727-739. doi: 10.1111/j.1601-183X.2012.00810.x

Zhao, M. L., and Wu, C. F. (1997). Alterations in frequency coding and activity dependence of excitability in cultured neurons of Drosophila memory mutants. J. Neurosci. 17, 2187-2199.

Zhong, Y., Budnik, V., and Wu, C. F. (1992). Synaptic plasticity in Drosophila memory and hyperexcitable mutants: role of cAMP cascade. J. Neurosci. 12, 644-651.

Zhong, Y., and Wu, C. F. (1991). Altered synaptic plasticity in Drosophila memory mutants with a defective cyclic AMP cascade. Science 251, 198-201. doi: 10.1126/science.1670967

Zhong, Y., and Wu, C. F. (2004). Neuronal activity and adenylyl cyclase in environment-dependent plasticity of axonal outgrowth in Drosophila. J. Neurosci. 24, 1439-1445. doi: 10.1523/JNEUROSCI.0740-02.2004

Conflict of Interest Statement: The author declares that the research was conducted in the absence of any commercial or financial relationships that could be construed as a potential conflict of interest.

Copyright $(9) 2015$ Lee. This is an open-access article distributed under the terms of the Creative Commons Attribution License (CC BY). The use, distribution or reproduction in other forums is permitted, provided the original author(s) or licensor are credited and that the original publication in this journal is cited, in accordance with accepted academic practice. No use, distribution or reproduction is permitted which does not comply with these terms. 